\title{
The Influence of Cooperative Game Design Patterns for Remote Play on Player Experience
}

\author{
Anastasiia Beznosyk, Peter Quax, Karin Coninx, Wim Lamotte \\ Hasselt University - tUL — IBBT \\ Expertise Centre for Digital Media \\ Wetenschapspark 2, B-3590 Diepenbeek (Belgium) \\ \{anastasiia.beznosyk, peter.quax, karin.coninx,wim.lamotte\}@uhasselt.be
}

\begin{abstract}
The collaborative nature of many modern multiplayer games raises a lot of questions in cooperative game design. We address one of them in this paper by analyzing cooperative game patterns in remote gameplay in order to define benefits and drawbacks for each one. With the help of a user experiment, we analyzed player experience in a set of existing cooperative patterns for games played remotely without communication. By comparing patterns, supporting closely- and looselycoupled collaboration, we discovered that the first type provided a more enjoyable experience but introduced additional challenges in case of a lack of communication. By analyzing patterns for both closely- and loosely-coupled interaction, we determined the most beneficial pattern within each type. We concluded with the results of a pattern comparison in colocated and remote setups.
\end{abstract}

\section{Author Keywords}

Cooperative game patterns; remote gameplay; communication; closely-coupled collaboration; loosely-coupled collaboration; player experience.

\section{ACM Classification Keywords}

H.5.2 Information Interfaces and presentation: User Interfaces; H.5.3 Group and Organization Interfaces: Evaluation/methodology.

\section{INTRODUCTION}

Integrating collaboration in some genres of multiplayer games has increased their popularity. Such games encourage players to team up and work together towards common goals whilst emphasizing group interest versus personal stakes. When creating a collaborative game, one has to motivate players to interact with each other in an effective manner. One possible solution is to incorporate different forms of interaction based on the coupling between players, either

Permission to make digital or hard copies of all or part of this work for personal or classroom use is granted without fee provided that copies are not made or distributed for profit or commercial advantage and that copies bear this notice and the full citation on the first page. To copy otherwise, or republish, to post on servers or to redistribute to lists, requires prior specific permission and/or a fee.

APCHI'12, August 28-31, 2012, Matsue-city, Shimane, Japan.

Copyright 2012 ACM 978-1-4503-1496-1/12/08...\$15.00. closely-coupled or loosely-coupled. While the first one implies one player's actions to be directly influenced by the others, the second form of interaction leaves more independence for the players.

Since collaboration has evolved from merely an additional element to a core component of a game, cooperative design has become an integral part of game design. Many game designers are currently exploring different ways of the integration and evaluation of cooperative activities within their games [6]. In doing so, one of the open issues remains the discovery and analysis of cooperative patterns that could improve interaction between players in multiplayer games with a collaborative nature. Additionally, as gaming occurs under a variety of circumstances (players can be located next to each other or play remotely, be able or not to hear and/or see partner, communicate through text chat,...), these varying conditions may directly or indirectly affect the player's experience. The same type of interaction could, therefore, result in a completely different player perception and influence his/her choice to play the game in the future. Most likely, the conditions a game is played in, affects closely-coupled interaction more due to a high dependency between players. Although a lot of cooperative games on the market are based on close coupling between players, most of them are designed for a co-located setup (e.g. Wii games ${ }^{1}$ ) or have facilities for rich communication. When it is not easy or even not possible to have this kind of continuous communication between players, closely-coupled interaction can suffer more from these setup conditions and, as a result, decrease player engagement with the game.

We investigate cooperative game patterns where gameplay occurs over a distance and players are not able to communicate in any form. We have performed a comparative analysis of several cooperative patterns. Although modern games usually apply a combination of patterns, in our study we look at separate patterns to define those best suitable for given conditions. Using different criteria, each pattern was graded based on its influence on player experience, revealing the drawbacks and benefits of each pattern. Six popular patterns were chosen $[6,10]$ based on their high frequency of occurrence in modern multiplayer cooperative games. Based on these findings, we define the best performing patterns separately for closely- and loosely-coupled collaboration. Finally, we illus-

\footnotetext{
${ }^{1}$ www.nintendo.com/wii
} 
trate the differences between the evaluation of the same patterns in both situations: when they are used remotely and colocated.

\section{RELATED WORK}

Game design patterns have been in focus for many years. Game patterns are descriptions of reoccurring interaction, that depict how game components are used by players to affect various aspects of gameplay [2]. In [2], the authors have identified a large amount of patterns by analyzing existing games, some of them being classified as the cooperative patterns with different possibilities and incentives for the players to achieve things together. In the last decades, cooperation has developed from an additional feature into a full-grown game component, motivating more and more players to join a game. Although several works attempted to address the question of cooperative game design patterns $[1,4,6,7,9,10,11]$, it remains open in the research community.

In [9], the cooperative nature of interaction between players is classified by the order in which actions may be taken: turntaking and simultaneous games. Zagal et al. [11] explore cooperative patterns based on board games. Interaction patterns in massive multiplayer games (based on the example of Star War Galaxies) are investigated by Ducheneaut et al. in [4], but here the authors focus on the social aspect of interaction (verbal and non-verbal communication between players). Cooperation in educative applications is studied by Holzinger et al. [8] and Ebner et al. [5]. Other studies [1, 7] investigate cooperative game design as a motivational factor for special target groups (e.g. people with disabilities) and focus on such patterns as enforced collaboration, sequentiallydependent roles, etc.

More elaborated research in the area of cooperative design patterns is performed in $[6,10]$. Following the same approach presented in [2], in [10], Rocha et al. define several cooperative design patterns by analyzing numerous commercial games, that support some form of collaboration between players. This list is considerably extended by El-Nasr et al. [6]. Here, the authors not only introduce a more complete series of cooperative game patterns, but also describe a methodology to evaluate the cooperative nature of games - Cooperative Performance Metrics (CPMs). Using this methodology, they analyze which pattern triggers which event during the shared gameplay (e.g. laughing, strategy discussion, etc.). The aim of the analysis was to investigate connections between the CPMs and the cooperative design patterns discussed in their study resulting in building better cooperative games.

The evaluation described in [6] was performed for several existing cooperative games that were played on a shared screen, and players could easily and naturally communicate with each other. The analysis of cooperative design patterns in a remote setup without communication has not yet been performed. To our knowledge, most cooperative games allow at least basic communication with the help of text chat, while some of them provide players with the ability to hear and see their partners. Nowadays, only a few games are available on the market where cooperation between players is supported without the ability to communicate in any form (e.g. Jour$n e y^{2}$ ). Therefore, we find it crucial to investigate how interaction between players can be improved based on introducing different cooperative patterns in order to compensate a possible negative effect due to a lack of communication.

\section{EXPERIMENT}

Our work evaluates cooperative game design patterns from the perspective of a remote setup where interaction between players happens without the possibility of directly communicating with one another. A user experiment was conducted, where players had to evaluate six custom games, each based on one of the cooperative patterns. For purposes of the experiment, a selection of patterns (selected from the list presented in [6]) was made based on their popularity and the frequency of appearance in existing multiplayer games. The goal of our study is two-fold. First, we analyze to what extent each cooperative pattern is appropriate for remote play without communication depending on a variety of criteria. Here, based on the coupling between players, we group the selected patterns either in a closely- or loosely-coupled type of interaction, in order to define the most benefiting patterns for each group. Secondly, a comparative analysis is performed between the same patterns when they are evaluated in co-located and remote setups.

\section{Participants}

Thirty-six unpaid subjects (thirty-one males and five females) participated in the experiment. Their average age was 28 years old, ranging between 21 and 38. Most of them had a computer science background and were recruited among university staff and students. Based on self evaluation, the average player experience with multiplayer games was 3.42 on a scale from 1 (never played) to 5 (played a lot).

\section{Setup}

During the experiment two players were located in neighboring rooms separated by a hallway. Each player used a 15.4" laptop connected over a LAN. One of the laptops was a HP Compaq 8510p (Intel Core 2 Duo T8100, $2.1 \mathrm{GHz}, 3 \mathrm{~GB}$ with ATI Mobile Radeon HD2600 graphic adapter) and the other was a Dell Latitude E6510 (Intel Core i3 M370, $2.4 \mathrm{GHz}$ 2 GB with NVIDIA NVS 3100M). A separate external keyboard was attached to each laptop for a more comfortable input. There was no communication possible between the two players. To avoid any chance of hearing each other, music was played in the background.

\section{Procedure}

Eighteen pairs of participants consecutively played six different collaborative games, described in detail in the following section. During each game the player had to collaborate with his/her partner who was located in a different room. Players were coupled anonymously and, therefore, did not know who their partner was. Any form of communication (voice chat, text chat, pop-up messages, etc.) was avoided. Pop-up windows were used only in one game to support some basic level of awareness between two players. Several days before

\footnotetext{
${ }^{2}$ www.thatgamecompany.com/games/journey
} 


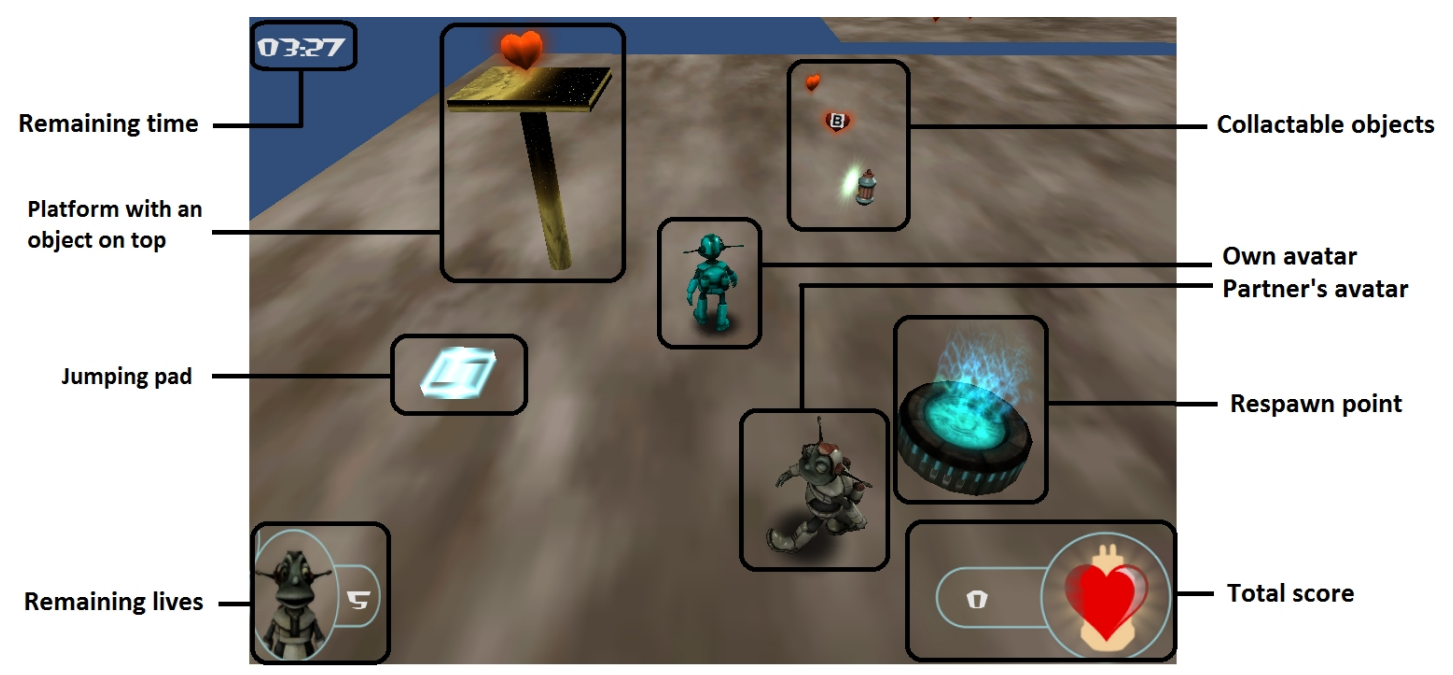

Figure 1. The gaming environment.

the actual experiment, a pilot test was performed to check the playability of every game.

Before the experiment, participants read a brief introduction and conducted a five minute trial to familiarize themselves with the gaming environment and controls. In addition, written rules were given, in which both the goal and the way of interacting with the partner were explained. After each game, players were asked to evaluate the subjective perception of their experience. In particular, they were asked to quantify the following aspects of collaboration:

- the necessity to discuss the game (strategy, ask your partner for help, etc.);

- level of collaboration with the partner;

- the amount of waiting for the other player during the game;

- level of awareness of the partner's actions;

- negative impact on the efficiency caused by the absence of communication, the physical distance, the necessity to wait for the other player, the actions of the other player;

- negative impact on the enjoyment caused by the absence of communication, the physical distance, the necessity to wait for the other player, the actions of the other player.

For evaluation purposes, a visual analogue scale (VAS) [3] was used. The participants marked on the $10 \mathrm{~cm}$ line the point that they felt represented their perception of the current state from not at all to very much. Additionally, the players ranked the six games based on the level of their enjoyment. It took approximately 60 minutes for each pair to complete the actual experiment.

\section{Games}

Six custom games were created for the experiment, each adopting one of the selected cooperative game patterns (Table 1). Based on the coupling between players, we classified each game into one of two categories: closely- or looselycoupled. If a game required a lot of waiting or if the actions of one player directly affected the other player, it was categorized as the first type. The games that did not require tight collaboration between players and allowed more independent performance were assigned to the second type.

For every game, a similar 3D virtual environment was developed, which consists of several islands (a rectangular area, on which all game elements are located). Players are represented by alien-like avatars used from Unity $3 \mathrm{D}$ tutorial $^{3}$. To distinguish the two avatars in the virtual environment, one is colored in a light blue color, while the other avatar is brown. Players are able to navigate freely in the environment and are not forced to stay together. They have to collect different objects by running over them. Some of the objects are located on higher platforms not directly reachable by the players. Therefore, they have to use the jumping pads that help a player to jump higher to collect certain items. In order to get on a different island players need to jump across the abyss. If one of the players falls off the island the team loses one life. After the fall, the player reappears at one of the respawn points. A group score is calculated and analyzed to measure the successfulness of the game completion. The game continues until one of the following conditions is met: (1) players collected all required objects; (2) players lost all their lives; (3) the time ran out. An example of the environment with all gaming elements is shown on Figure 1.

For closely-coupled Game 1, a limited resources pattern is adopted. Two players have to collect items, but are able to store a maximum of 10 items at the same time. Once both players reach the maximum amount of items, they can collect the following 10 objects. If one of the players collects 10 objects he/she has to wait for the other player and cannot pick up new items in the meantime.

In closely-coupled Game 2, a complementary pattern is used, which implies that players have a different role to complement each others' activities within the game. During this

\footnotetext{
${ }^{3}$ available online at http://unity3d.com/support/resources/tutorials/3dplatform-game
} 


\begin{tabular}{|c|l|c|c|c|c|c|c|c|}
\hline $\mathbf{N r}$ & Design pattern & $\begin{array}{c}\text { Closely- or loosely- } \\
\text { coupled }\end{array}$ & $\begin{array}{c}\text { Amount of } \\
\text { islands }\end{array}$ & $\begin{array}{c}\text { Amount of } \\
\text { lives }\end{array}$ & $\begin{array}{c}\text { Total amount } \\
\text { of objects }\end{array}$ & $\begin{array}{c}\text { Necessary } \\
\text { to pick up }\end{array}$ & $\begin{array}{c}\text { Object } \\
\text { type }\end{array}$ & $\begin{array}{c}\text { Time, } \\
\text { min }\end{array}$ \\
\hline 1 & Limited resources & Closely-coupled & 5 & 5 & 100 & 75 & hearts & 7 \\
\hline 2 & Complementary & Closely-coupled & 5 & 5 & 100 & 75 & hearts & 7 \\
\hline 3 & $\begin{array}{l}\text { Interaction with } \\
\text { the same object }\end{array}$ & Closely-coupled & 4 & 5 & 100 & 75 & hearts & 7 \\
\hline 4 & Shared puzzles & Loosely-coupled & 5 & 5 & 200 & 10 & letters & 5 \\
\hline 5 & $\begin{array}{l}\text { Abilities that can } \\
\text { be used on other } \\
\text { players }\end{array}$ & Loosely-coupled & 4 & 5 & 200 & 150 & $\begin{array}{c}\text { hearts } \\
\text { weapons }\end{array}$ & 5 \\
\hline 6 & Shared goals & Loosely-coupled & 5 & 3 & 150 & 115 & hearts & 5 \\
\hline
\end{tabular}

Table 1. Cooperative games used during the experiment.

game two roles are introduced. One player moves the jumping pad around the island while not being able to jump, and the other player uses it for jumping to reach objects located on higher platforms. There is only one jumping pad on each island. The roles are assigned randomly when players start the game.

Closely-coupled Game 3 follows an interaction with the same object pattern. In this game players have to move the jumping pad simultaneously. As soon as one of the players selects the jumping pad to move, the other player receives a message on his/her screen that the pad is selected and he/she is needed to help moving it. However, it does not indicate the location where the player has to be in order to help his/her teammate. When selected by two players, the jumping pad can be moved when both players walk in the same direction. Both players can use it for jumping. Similar to game 2, there is only one jumping pad on each island.

Loosely-coupled Game 4 utilizes a shared puzzles pattern. Here, the focus is to collect 10 special objects: each contains a heart with a letter on one side. Once all 10 special objects and therefore ten letters are found, players need to use them to formulate a word containing all the letters, and put them in a designated window. The game succeeds when the word is entered correctly. Players do not see what words are entered by their partners while guessing. Once the correct solution is given by one player, the other one can also see it in his/her window.

An abilities that can be used on other players pattern is used in loosely-coupled Game 5. In this game, players have to collect two types of objects: hearts and weapons. Each one is assigned to one player. They can see only one type which is randomly assigned on starting the game. Every time a player collects his/her 10 items, he/she gets the ability to see the partner's objects for about twenty seconds, and is able to collect them as well. The goal of the game is not only to collect enough objects as a team. Individual results of each player also need to exceed a certain amount of items of both types.

Loosely-coupled Game 6 utilizes a shared goals pattern. The collaboration is reduced to a shared goal of collecting a certain amount of objects while acting independent from the partner.

\section{Design}

During the experiment, a within-subject design was used. The independent variable was the game type with six conditions. All participants, in pairs, had to complete six sessions testing every game type. The order of the conditions was counterbalanced using a balanced Latin square design. The dependent variables were: task completion time, player score, total group score and amount of lives lost. A subjective evaluation of each game type was collected through a self-developed post-experiment questionnaire.

\section{RESULTS}

This section presents the results of our study. As mentioned earlier, the main goal of the experiment was to analyze the most popular cooperative game design patterns in a remote setup with no communication. We aimed to investigate which patterns, if any, were affected more than the others by the conditions they were played in. First, we present an overview of player performance and preference. Secondly, we report results that reflect an impact of the remote setup on the player experience (absence of communication and physical distance). Finally, the patterns are compared from the perspective of collaboration between players. Here, we also investigate if any relation between player performance and his/her partner's evaluation exists. Throughout this section, we name games by the corresponding number. For detailed information on each game we refer to Table 1.

\section{Player Performance and Preference}

Table 2 summarizes the results of player performance (game completion rate and mean player efficiency) and preference (enjoyment and player decision upon games' suitability).

\section{Performance}

For every game, players had to collect a certain amount of objects within a certain time limit in order to successfully complete it. In order to compare three closely-coupled games, they all had equal conditions: players had to pick up 75 objects within 7 minutes (Table 1). Conditions of looselycoupled games varied due to differences in game design for these patterns. Due to the different ways of interacting between players, some games were found to be more difficult to finish within a given period. Analysis of the game completion rate has revealed that the majority of players managed to finish only two games in time: the ones with the limited resources and shared goals patterns (Table 2). As can be seen, 


\begin{tabular}{|c|c|c|c|c|c|}
\hline$\#$ & $\begin{array}{c}\text { Completion } \\
\text { rate, \% }\end{array}$ & $\begin{array}{c}\text { Average amount } \\
\text { of collected objects }\end{array}$ & $\begin{array}{c}\text { Efficiency, } \\
\text { obj/min }\end{array}$ & $\begin{array}{c}\text { Enjoy- } \\
\text { ment }^{*}\end{array}$ & $\begin{array}{c}\text { Not suitable } \\
\text { rate, \% }\end{array}$ \\
\hline 1 & 88.89 & 74.22 & 8.54 & 2 & 11.11 \\
\hline 2 & 11.11 & 56.06 & 4.04 & 5 & 16.67 \\
\hline 3 & 0 & 54.61 & 3.93 & 6 & 19.44 \\
\hline 4 & 16.67 & 109.28 & 11.10 & 3 & 19.44 \\
\hline 5 & 38.89 & 144.56 & 16.40 & 4 & 11.11 \\
\hline 6 & 83.33 & 111.94 & 16.41 & 1 & 13.89 \\
\hline
\end{tabular}

Table 2. Player performance and preference.

the difference between the average amount of collected objects and the necessary amount of objects for games 2 and 3 was higher than in the other games.

Due to our decision to design games with equal conditions, in most cases the games were not completed successfully but finished because of a lack of time. In these conditions it was hardly possible to compare game completion time. Therefore, as an alternative measurement of performance, we calculated the efficiency of the players for every game. It was defined as the amount of objects collected by each player in one minute. Repeated measures ANOVA has shown a significant difference across six patterns $(F(2.73,95.37)=105.37, p<0.01)$. A Bonferroni post-hoc test has demonstrated a significantly lower efficiency in two of the closely-coupled games, games 2 and 3 , than in any other game $(p<0.01)$. At the same time, no significant difference was found between the three loosely-coupled games, all of them sharing a high level of player efficiency.

\section{Preference}

The participants were asked to rank the six games according to the level of their enjoyment. They were explicitly asked to judge each game based on the way in which players interact and not take into account other gaming elements (e.g. design of the environment, look of the avatar, etc.). We have analyzed how many times each game appeared on the first, second, etc. and last places, in order to define the most preferred games. Game 3 has been found to be the most enjoyable, followed by games 2, 5, 4 and 1. Game 6 has been indicated as the least enjoyable due to its total independence between players while working towards a shared goal. In this game, the other player was often considered as a non-player character or competitor than a team member. From Table 2 we observe that the low efficiency in closely-coupled games (games 2,3) did not decrease player enjoyment.

Finally, we asked players to define if there were any game patterns that are not suitable for playing remotely without communication. Participants were free to indicate which game(s), to their opinion, they would prefer not to play under the same circumstances. It was also allowed to leave the question unanswered if they found them all suitable. Although we have observed that participants found games 3 and 4 to be the least suitable (possible reasons for that will be shown further throughout the Results section), a high percentage of participants indicated all games to be playable, even when there was no communication.

\section{Impact of the Remote setup on Player Experience}

Once the level of player performance and enjoyment for each game was evaluated, we aimed to estimate to what extent they were affected by the setup conditions (the absence of communication and physical distance).

\section{Absence of Communication}

First of all, we asked players to evaluate to what extent they felt a necessity to discuss the game. We considered any type of topic that could improve gameplay, e.g. strategy discussion, asking for help, etc. Our findings (Figure 2(a)) tend to confirm the general expectation that loosely-coupled games require less communication. Most of them have been evaluated significantly lower by the participants than closelycoupled games. Repeated measures ANOVA has shown a significant difference between six games $(F(5,175)=11.45$, $p<0.01)$. A Bonferroni post-hoc test has demonstrated that game 6 required significantly less communication $(p<0.01)$ than all other games (except game 5). It has been also discovered that games 2 and 3 required more discussion than game 5 . At the same time, there was no difference between closelycoupled games.

Observing these necessity ratings (indicating an eagerness to communicate within certain games), it is important to see how the lack of communication affects player enjoyment and efficiency. As can be seen from Figure 2(b), for all games players indicated a perceived negative impact of communication on their enjoyment as relatively low (on average for every game, it did not exceed 5 on the 0 to 10 scale). A repeated measures ANOVA has shown a significant difference across the games $(F(5,175)=6.13, p<0.01)$, but a Bonferroni post-hoc test has indicated that this difference in fact only existed between game 2 and games 5 and 6 .

Finally, we analyzed whether or not a lack of communication decreased player efficiency. Figure 2(b) shows that games 2 and 3 are most affected. Players indicated that they felt they could be more efficient if they were able to discuss the strategy. Repeated measures ANOVA $(F(5,175)=14.82, p$ $<0.01)$ and a follow-up Bonferroni post-hoc test $(p<0.01)$ confirmed that this difference was significant. Moreover, we found that even among loosely-coupled games game 4 was rated significantly worse $(p<0.01)$. For this particular game, players found communication important as it would improve the word guessing assignment if they would be able to discuss the possible options with a partner. 


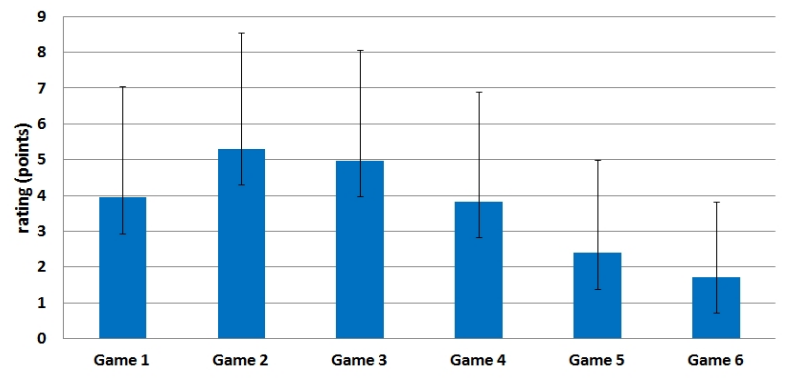

(a)

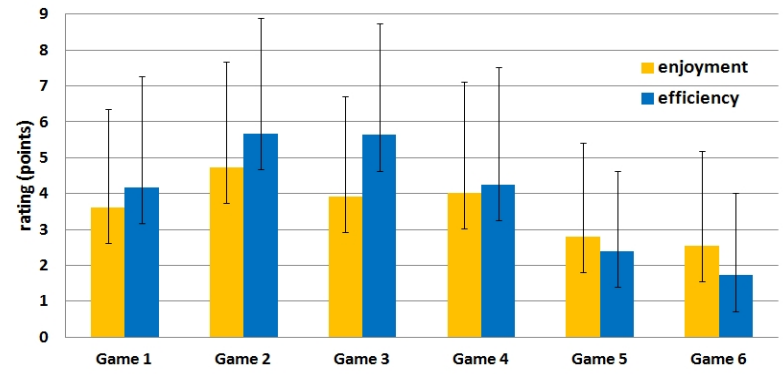

(b)

Figure 2. Necessity to discuss the game (a) and its influence on player enjoyment (yellow) and efficiency (blue) (b).

\section{Physical Distance}

Furthermore, we evaluated the negative impact caused by the physical distance between players. For both enjoyment and efficiency, players indicated that the physical distance had a rather low negative effect (Figure 3). For its impact on player enjoyment, we did not find any significant difference between the six games $(F(3.33,116.41)=2.48, p>0.05)$, indicating that all games were equally enjoyable when played over distance. When analyzing the influence on player efficiency, we found a significant difference $(F(3.71,129.96)=$ $3.7, p<0.01$ ). Further post-hoc testing showed that these difference existed only between games 3 and $6(p=0.021)$. No difference between games within the closely-coupled and loosely-coupled groups was found.

\section{Impact of the Type of Collaboration on Player Experience} The final part of the analysis is focused on the collaborative aspects of each game. Every game was developed in such a way that it follows a certain cooperative design pattern. Although all patterns are assumed to support team work, the level of collaboration in every game may be perceived differently among players.

\section{Level of Collaboration}

When creating multiplayer games, in some cases game designers try to insert tasks that require a high level of collaboration between players in order to enhance their gaming experience. Analyzing the six patterns presented in this paper, our findings confirmed that closely-coupled games tended to provide a higher level of collaboration than loosely-coupled. All games (except game 5) were significantly more collaborative

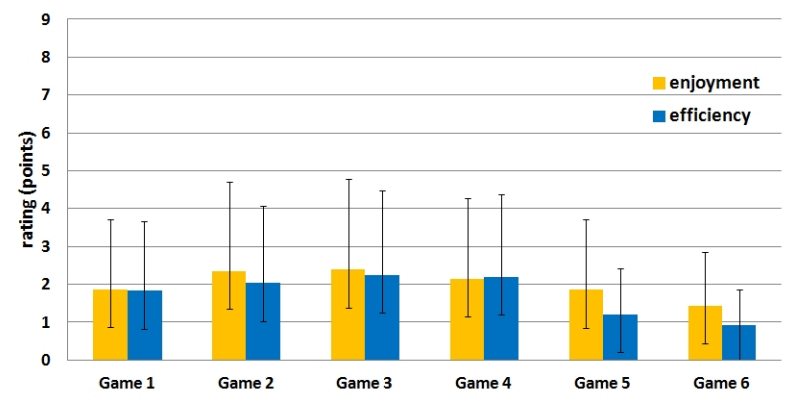

Figure 3. Influence of physical distance on player enjoyment (yellow) and efficiency (blue). than game $6(F(3.47,121.58)=77.09, p<0.01)$, with games 2 and 3 showing the highest level of collaboration (Figure 4). Therefore, we can state that among both closely-coupled and loosely-coupled games, some patterns can be more efficient when it comes to providing high level of collaboration between players.

Additionally, we wanted to check for which types of games a higher level of collaboration will result in a higher gaming enjoyment. We found a positive correlation between collaboration and level of player enjoyment for games $1(R=0.39, p$ $=0.019), 2(R=0.36, p=0.031)$ and $4(R=0.39, p=0.018)$. Although game 3 had one of the highest levels of collaboration, it did not show an increase in player enjoyment. One possible explanation for this is that the other factors (e.g. way of interacting between players during the game) influenced player preference towards this game more than the level of collaboration.

\section{Waiting for Others}

One of the characteristics, typical for team work, is the amount of time players have to wait for each other before being able to continue. The waiting time is caused by a variety of reasons: player skills, occupation with different ingame tasks, etc. Therefore, we wanted to investigate how often it was necessary to wait for the partner in each game. As we did not allow communication between players, we expected closely-coupled games to introduce more waiting time. This assumption was confirmed by further analysis. We asked players to estimate the necessity to wait for each other in every game. Figure 5(a) represents the amount of waiting between six test conditions. A significant difference

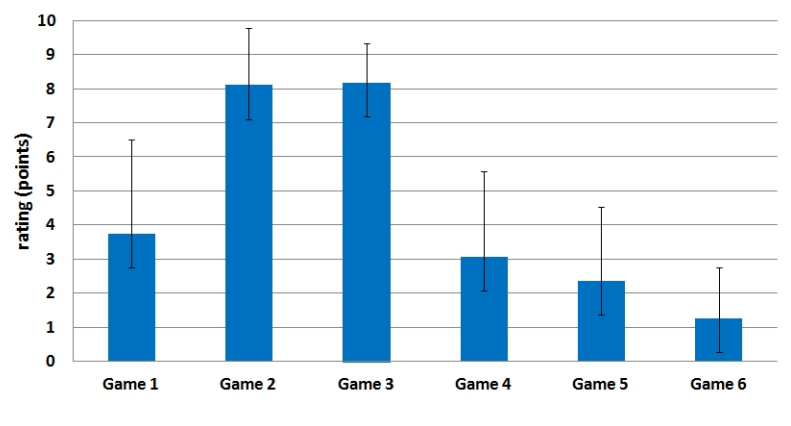

Figure 4. Level of collaboration. 
between them was found $(F(5,175)=37.79, p<0.01)$. A post-hoc analysis has shown that during any of the closelycoupled games, players spent significantly more time waiting for their partners than during the loosely-coupled gaming sessions. We observed that this was mainly due to the amount of collaboration required in closely-coupled games, although a difference in the experience level of players could also increase waiting time. At the same time no difference within the group of both closely- and loosely-coupled games was found.

As waiting time is a factor that can decrease player enjoyment and performance, we analyzed how their enjoyment and efficiency were affected (Figure 5(b)). Results of repeated measures ANOVA followed by a post-hoc test showed that player experience in closely-coupled games suffered significantly more from the necessity to wait $(F(3.09,108.21)=$ $16.85, p<0.01$ for enjoyment and $F(3.24,113.44)=26.43$, $p<0.01$ for efficiency).

Based on the subjective measurements, the necessity to wait decreased player efficiency and enjoyment. However, we still wanted to see whether or not the waiting time affected the player experience objectively, based on the actual performance. This is especially important in case of closelycoupled games as they introduce lots of waiting into the play. Surprisingly, the additional waiting in closely-coupled games did not affect players' efficiency, as no correlation was found. The same result was obtained for loosely-coupled games.

Additionally, we checked if there was any negative impact of waiting on the level of collaboration. A significant negative correlation between collaboration and amount of waiting time for game 3 was found ( $R=-0.33, p=0.047$ ), making it weaker among other closely-coupled games when it comes to providing high level of collaboration, as this game required quite a lot of waiting between players (players may move away from each other but need to combine their efforts in order to move the jumping pad).

\section{Influence of Partner's Actions}

In multiplayer games, the actions of one player often directly or indirectly affect the gaming experience of others. Here, we present results regarding the extent of the influence of the partner's action and behavior on the player's own performance and enjoyment. We expect that, when tightly collab- orating, players notice other players actions more, and therefore, are more affected by them.

One of our initial assumptions was that partner performance greatly affects player enjoyment, especially in closelycoupled games where two players are almost always working together. Repeated measures ANOVA rejected our assumption, indicating that the partner's actions did not decrease player enjoyment in certain games more than the others $(F(3.60,125.83)=2.63, p>0.05)$. Also, the values for negative effect of partner's action on player enjoyment provided by participants, remained very low (on average for every game, they did not exceed 3.5 on the 0 to 10 scale).

At the same time, it was not the case when comparing the influence of partner's actions on player efficiency. We found that closely-coupled games were affected more than looselycoupled games $(F(3.77,131.8)=14.95, p<0.01)$. As before, we have also checked the correlation between this influence and player enjoyment. No game showed a relation, indicating all games to be enjoyable even when the player efficiency was affected by the actions of the partner.

Finally, we wanted to see if the actual player contribution had an influence on the evaluation of the partner. We estimated player contribution to be based on the amount of objects he/she and his/her partner collected (total amount of objects collected by a team is considered as $100 \%$ ). Here, we confronted the player evaluation with the objective contribution of the partner to see if any correlation between those two existed. Such correlation existed only for games 2 and 3 (Table 3). In case of game 2, we found that higher partner's contribution reduced the negative effect of lack of communication on player enjoyment. In case of game 3, both player enjoyment and performance were related to the partner's contribution. The higher the contribution, the more it reduced a negative impact on player experience that existed due to the gaming conditions (i.e. lack of communication, physical distance and waiting). This allows us to conclude that tasks triggering a higher player performance may improve the outcome of team work. Based on the obtained results, we can, for instance, see that a higher player contribution can compensate for the negative effect caused by the setup conditions.

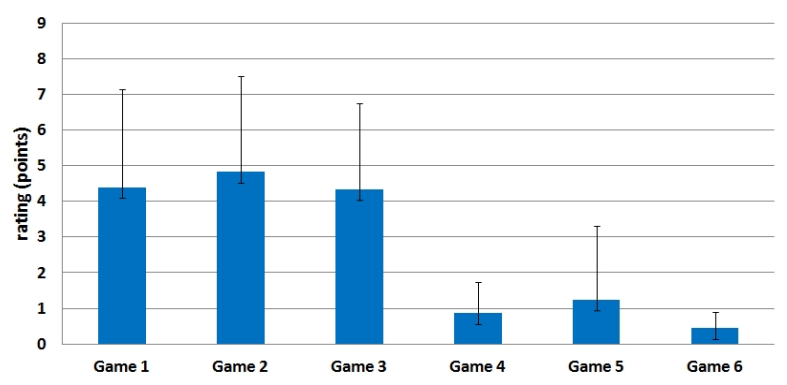

(a)

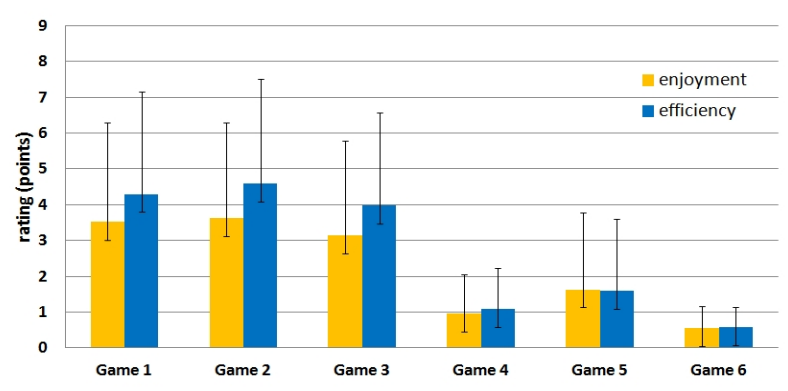

(b)

Figure 5. Necessity to wait for the partner (a) and its influence on player enjoyment (yellow) and efficiency (blue) (b). 


\begin{tabular}{|l|c|c|c|c|}
\hline & $\begin{array}{c}\text { Partner's contribution } \\
\text { \& enjoyment decreased } \\
\text { by lack of } \\
\text { communication }\end{array}$ & $\begin{array}{c}\text { Partner's contribution } \\
\text { \& effectiveness decreased } \\
\text { by lack of } \\
\text { communication }\end{array}$ & $\begin{array}{c}\text { Partner's contribution } \\
\text { \& enjoyment decreased } \\
\text { by physical } \\
\text { distance }\end{array}$ & $\begin{array}{c}\text { Partner's contribution } \\
\text { \& effectiveness decreased } \\
\text { by waiting }\end{array}$ \\
\hline Game 2 & $\begin{array}{c} \\
\\
\text { Game 3 }\end{array}$ & $p=-0.38$ & & \\
& $R=0.021$ & $R=-0.35$ & $R=-0.35$ & $R=-0.34$ \\
$p=0.018$ & $p=0.039$ & $p=0.039$ & $p=0.046$ \\
\hline
\end{tabular}

Table 3. Influence of partner's contribution.

\section{DISCUSSION}

To our knowledge, previous research initiatives have analyzed cooperative game patterns only when the players were present on the same location, and could naturally communicate and see each other [6]. When considering interaction over distance, especially when no communication is allowed, additional challenges are present when trying to maintain the same level of player experience. Therefore, games based on the same cooperative patterns can result in an entirely different experience when considered in a non-collocated setup. Knowing these differences may help game developers improve player interaction in collaborative multiplayer games, both for co-located and remote play.

In order to see how different patterns affect player experience under two different circumstances, we compared results obtained in our study with the evaluation of patterns provided in [6]. In their work, the authors have chosen four popular multiplayer collaborative games and defined a set of patterns that occur in each game. Then, using some self-developed Cooperative Performance Metrics (CPM), they mapped CPM elements to the cooperative patterns that caused them. Although we have not applied CPM for evaluation during our experiment, we believe some of the questions in our postexperiment questionnaire can be matched to certain CPM components. We had to eliminate game 1 from the comparison analysis as this pattern was not evaluated in [6].

The first metrics in [6] was 'laughter and excitement'. The authors reported that shared goals (in our case, game 6), shared puzzles (Game 4) and complementary (game 2) patterns provided the highest values for this metric, while abilities on other players (game 5) and interaction with shared object (Game3) caused less positive emotions. Although in our case we did not analyze laughter and excitement directly, we believe that it is comparable with the rating of games based on the player enjoyment. Oppositely to co-located play, in our case game 6 was found to be the least enjoyable, while games 2 and 3 had the highest preference. Based on our findings, we believe that such difference between remote and co-located setups is caused by the inability to talk. Some players even indicated that without communication, game 6 tended to be more competitive than collaborative, as one barely notices the other player, and tasks are performed individually.

The next CPM components were 'worked out strategies' and 'helping events'. These two patterns are directly connected with an ability to communicate between players. If you can communicate, you can easily decide upon the strategy or ask your partner for help. Therefore, we associated these two cri- teria with the evaluation of necessity to communicate. During the co-located condition the authors observed that patterns of shared puzzles (game 4) and goals (game 6) caused most of 'worked out strategies' and 'helping events'. These are followed by complementary (game 2) and interaction with same object (game 3) patterns. In our case, complementary and interaction with the same object patterns required the most communication between players. One of the reasons for that was players not willing to stay together in the gaming environment. As a consequence, once help was needed players could not immediately find their partner.

In their work, El-Nasr et al. analyzed the 'wait for each other' events that were caused by each pattern. In their analysis complementary (game 2) and interaction with same object (game 3) patterns did not cause waiting events at all. It is quite natural when you are together to immediately communicate what you are going to do, and whether it involves the other player. At the same time they have ascertained that shared puzzles (game 4) and goals (game 6) patterns were resulting in additional waiting. In our case due to their looselycoupled nature games 4 and 6 required the least waiting in comparison with game 2 and 3.

The final metric used in [6] was 'got in each others' way', which is similar to the influence of partner's actions in our questionnaire. Similarly to their results we observed that shared goals pattern (game 6) had the least influence of partner's actions, while the complementary pattern (game 2) was rated highest in both studies.

\section{CONCLUSION AND FUTURE WORK}

In this paper we have presented a user experiment where different types of collaboration were analyzed in a remote setup without communication between players. Six collaborative games were developed, each following one of the cooperative game design patterns: limited resources, complementary roles, interaction with the same object, shared puzzles, abilities that can be applied on other players and shared goals. Based on the coupling between players, each pattern was referred to as either closely-coupled or loosely-coupled.

Among closely-coupled games, complementary and interaction with the same object patterns were found to be the most enjoyable and provided the highest level of collaboration among all six patterns. At the same time, they were most affected by the lack of communication between two players. The game based on limited resources pattern was less influenced by the absence of communication and the remote setup, 
but among all closely-coupled games it showed the lowest level of collaboration and enjoyment.

When comparing loosely-coupled collaboration, the abilities that can be used on other players pattern showed a high level of player satisfaction with a minor negative effect brought by the lack of communication. This game did not provide players with a high perception of team work, but this did not impact player enjoyment. Absence of communication affected the shared goals pattern the least, but the game based on this pattern introduced the lowest level of enjoyment with (almost) no collaboration.

With this study we have made the first attempt to evaluate cooperative game patterns in remote setup where any form of communication was not supported. The evaluation of each pattern was based on a single game. Although we realize that the same game patterns can be integrated and designed in multiple ways, we believe that these results can be valid for further research in cooperative game design. Results from this study revealed several interesting design lessons that can be further applied in order to build better cooperative games being played under different circumstances or certain limitations (in our case, absence of communication).

We have covered only six cooperative game patterns. Analysis of other existing patterns is still necessary. Furthermore, an effective way of combining these patterns together should be investigated under different play conditions (remote or colocated, with or without communication). Defining what patterns work best together under different circumstances could be of significant value for game designers aiming for an increasingly enjoyable player experience.

\section{ACKNOWLEDGMENTS}

The research described in this paper is directly funded by Hasselt University through the BOF framework. The authors would like to thank Tom De Weyer for his assistance with the implementation and all participants who contributed to this research by taking part in the experiment.

\section{REFERENCES}

1. Battocchi, A., Pianesi, F., Tomasini, D., Zancanaro, M., Esposito, G., Venuti, P., Ben Sasson, A., Gal, E., and Weiss, P. L. Collaborative puzzle game: a tabletop interactive game for fostering collaboration in children with autism spectrum disorders (asd). In Proc. ITS 2009, ACM (2009), 197-204.

2. Björk, S., and Holopainen, J. Patterns in Game Design (Game Development Series). Charles River Media, Inc., 2004.

3. Crichton, N. Information point: visual analogue scale (VAS). Clinical Nursing 10, 5 (2001), 697 - 706.

4. Ducheneaut, N., and Moore, R. J. The social side of gaming: a study of interaction patterns in a massively multiplayer online game. In Proc. CSCW 2004, ACM (2004), 360-369.

5. Ebner, M., and Holzinger, A. Successful implementation of user-centered game based learning in higher education: An example from civil engineering. Computers \& Education 49, 3 (2007), 873 - 890.

6. El-Nasr, M. S., Aghabeigi, B., Milam, D., Erfani, M., Lameman, B., Maygoli, H., and Mah, S. Understanding and evaluating cooperative games. In Proc. CHI 2010, ACM (2010), 253-262.

7. Goh, W.-B., Fitriani, Ting, L. G., Shou, W., Goh, C.-F., Menon, M., Tan, J., and Cohen, L. G. Potential challenges in collaborative game design for inclusive settings. In Proc. of workshop on UI Technologies and Their Impact on Educational Pedagogy, available online at http://www.dfki.de/EducationCHI2011/(2011).

8. Holzinger, K., Lehner, M., Fassold, M., and Holzinger, A. Archaeological scavenger hunt on mobile devices: from education to e-business: A triple adaptive mobile application for supporting experts, tourists and children. In Proc. ICE-B 2011, INSTICC (2011), 131-136.

9. Pape, S., Dietz, L., and Tandler, P. Single display gaming: examining collaborative games for multi-user tabletops. In Proc. of workshop on Gaming Applications in Pervasive Computing Environments (2003).

10. Rocha, J. B., and Mascarenhas, S. P. R. Game mechanics for cooperative games. In ZDN Digital Game (2008), 73-80.

11. Zagal, J. P., Rick, J., and Hsi, I. Collaborative games: lessons learned from board games. Simul. Gaming 37, 1 (2006), 24-40. 